Cite this: Phys. Chem Chem. Phys. 2015, 17, 20873

Received 1st June 2015 Accepted 17th July 2015

DOI: $10.1039 / \mathrm{c} 5 \mathrm{cp} 03147 \mathrm{~h}$

www.rsc.org/pccp

\section{Thin films of size-selected Mo clusters: growth modes and structures}

\author{
Andreas Dollinger, ${ }^{a}$ Eun Ji Park, ${ }^{b}$ Christoph H. Strobel, ${ }^{a}$ Hannes Bleuel, ${ }^{a}$ \\ Alexander Marsteller, ${ }^{a}$ Hyun Ook Seo, ${ }^{c}$ Young Dok Kim ${ }^{\star b}$ and Gerd Ganteför*a
}

\begin{abstract}
Thin films of $\mathrm{MoO}_{3}$ were prepared by deposition of size-selected ligand-free Mo clusters under high vacuum conditions and subsequent exposure to air. The growth pattern is highly dependent on the cluster size. At low coverage, small clusters $\left(\mathrm{Mo}_{51}\right)$ form a continuous monolayer of fused particles. On top of this monolayer, additional clusters survive as individual entities. Medium sized clusters $\left(\mathrm{Mo}_{251}\right.$ and $\mathrm{Mo}_{1253}$ ) do not coalesce and form a monolayer of clusters. Close examination using in situ scanning tunneling microscopy reveals a local order of the particles. At higher coverage a new pattern of large 3-dimensional aggregations of clusters (pylons) appears. The pylons are not formed under high vacuum conditions. Their formation is most likely caused by the air exposure. For the largest clusters (Mo 3349 ) studied here, no monolayer is formed. Instead, the clusters are randomly distributed as expected for particles with zero mobility. These results demonstrate the high potential of cluster deposition for the production of new types of nanostructured surfaces, thin films and nanomaterials.
\end{abstract}

\section{Introduction}

The growth of high quality thin films by chemical and physical vapor deposition is one of the key challenges of the semiconductor and electronic device industry. ${ }^{1,2}$ Furthermore, thin films are used as oxidation protection, as hard-coating and for self-cleaning surfaces. ${ }^{2,3}$ The physical and chemical properties of a thin film on a substrate are determined by the growth mode. Therefore, much attention has been paid to the understanding of the microscopic mechanism of the growth modes of thin films. ${ }^{4}$ In general, there are three main growth modes. A layer-by-layer growth is observed when the lattice mismatch between the substrate and the thin film is small and the adhesion forces between the atoms in the thin film and between the atoms and the substrate are comparable (Frank-van-derMerwe growth). If the interaction between the substrate and the thin film is weak, the growth of 3-dimensional (3-D) islands is observed (Volmer-Weber). The Stranski-Krastanov growth mode corresponds to an intermediate between the first two cases. The completion of the first monolayer is followed by the growth of 3-D island. ${ }^{4}$

Nanoparticles are different from the respective bulk materials because of their high surface-to-volume ratio. The high abundance

\footnotetext{
${ }^{a}$ Department of Physics, University of Konstanz, D-78457 Konstanz, Germany. E-mail: gerd.gantefoer@uni-konstanz.de

${ }^{b}$ Department of Chemistry, Sungkyunkwan University, 440-746 Suwon, Republic of Korea. E-mail: ydkim91@skku.edu

${ }^{c}$ Center for Free-Electron Laser Science/DESY, D-22607 Hamburg, Germany
}

of under-coordinated atoms on their surface gives rise to unusual chemical properties, which are exploited in heterogeneous catalysis. ${ }^{5}$ For example, Au nanoparticles smaller than 3-4 $\mathrm{nm}$ are active catalysts for various reactions such as the CO oxidation, whereas the bulk counterpart is inert. ${ }^{5-9}$ In addition, quantum confinement effects and unique geometric structures dissimilar to the bulk counterparts result in unexpected electronic properties, ${ }^{8-14}$ which make clusters interesting for future nano-electronics.

In principle it is possible to synthesize not only thin films, but also new cluster-based materials from size-selected clusters. However, nanoparticles and clusters are generally unstable due to the high surface-to-volume ratio and therefore, adjacent nanoparticles or clusters easily agglomerate and form larger entities. ${ }^{15,16}$ Ligand shells may protect the particles from coalescence. ${ }^{17}$ However, such shells isolate the clusters from the environment and for several applications such as in catalysis bare clusters are required. Only very few examples of materials built from ligand-free clusters are known. These are clusters with a high stability such as the fullerenes and $\mathrm{Sb}_{4}$. From these "magic" clusters new materials have been successfully synthesized and it is shown that indeed the particles survive as individual entities. ${ }^{18-22}$ In gas phase studies, many more "magic" clusters with stable electronic and geometric configurations have been identified. All these magic clusters might be building blocks for new cluster materials. However, only very few attempts have been made to actually synthesize such materials. ${ }^{12,13,23-25}$ There is an experimental difficulty: ligand-free size-selected clusters can only be produced in very small amounts. It is enough for 
spectroscopic studies of individual species in the gas phase, but it is not enough to accumulate more than a monolayer. Here, we use a high intensity cluster source with subsequent mass selection to overcome this problem. The setup allows the preparation of films with a thickness of several 100 nanometers. As we will show below, the growth modes of thin films of clusters differ significantly from those of atoms.

\section{Experimental}

The experimental set-up has been described in detail elsewhere. ${ }^{22}$ In short, $\mathrm{Mo}_{n}{ }^{-}$cluster anions are produced via magnetron sputtering, mass-selected with a sector magnet (mass-resolution $m / \Delta m=20$ ) and deposited within the soft-landing criterion at highvacuum conditions $\left(p<1 \times 10^{-8}\right.$ mbar $)$ on highly ordered pyrolytic graphite (HOPG). Due to the limited mass resolution in all experiments a range of slightly different masses is deposited. The width of the mass distribution is $\pm 5 \%$ around the nominal mass.

On a freshly deposited sample there are places with different cluster coverage. The cluster ion beam is focused to the middle of the sample. There, the thickness of the film typically exceeds $100 \mathrm{~nm}$. Off the center, the coverage decreases and at the fringe of the deposition spot the coverage is below a cluster monolayer. By recording microscopic images of different spots, the coverage dependence of the thin film morphology can be determined while studying a single sample.

After deposition, the samples are transferred to a scanning tunneling microscope (STM, “OMICRON VT-STM”) under highvacuum conditions. They are not exposed to air. The topography of the cluster films is investigated in the constant current mode using PtIr tips. In addition, in situ X-ray photoelectron spectroscopy (XPS) studies are performed.

The topography of the surfaces is further investigated by ex situ atomic force microscopy (AFM). The AFM is operated under air in the intermittent contact mode (tapping mode). After exposure to air, the samples are characterized again with XPS. All experiments are conducted at room temperature.

\section{Results and discussion}

\subsection{Results of the in situ studies}

Our ultimate goal is the study of cluster thin films at ambient conditions, because this could be important for applications. Before we perform ex situ studies under air, thin films are prepared under high vacuum conditions. By comparison with the ex situ data information about the structural change driven by the air exposure can be gained.

Fig. 1 shows in situ XPS Mo $3 \mathrm{~d}_{5 / 2}$ and $3 \mathrm{~d}_{3 / 2}$ core level spectra of Mo clusters deposited on HOPG. Each core level spectrum is de-convoluted into different oxidation states of $\mathrm{Mo}^{27}{ }^{27}$ or $\mathrm{Mo}_{51}$, the clusters are almost completely oxidized into $\mathrm{Mo}(\mathrm{Iv})$ and $\mathrm{Mo}(\mathrm{v})$. I.e., most of the clusters are already significantly oxidized by the residual gases under high vacuum conditions (Fig. 1a). Obviously, the small clusters are highly reactive. In the XPS spectra of the larger clusters $\left(\mathrm{Mo}_{251}, \mathrm{Mo}_{1253}\right.$, Mo $\left.{ }_{3349}\right)$ metallic Mo dominates (Fig. 1b-d). The larger clusters are chemically less reactive.

Fig. 2 and 3 display in situ STM images of deposited Mo clusters with different sizes. For the smallest clusters $\left(\mathrm{Mo}_{51}\right)$ studied here, no STM images with sufficient quality could be recorded. It is possible that these small clusters are too mobile to allow STM imaging with high resolution. Another possibility is that these clusters fused into a compact thin film. For the medium sized clusters $\left(\mathrm{Mo}_{251}\right)$, 2-dimensional islands are formed (Fig. 2a). In these islands individual species can be discerned (Fig. 2b). A close inspection of Fig. $2 b$ reveals that the clusters form a locally hexagonal arrangement. The reciprocal pattern obtained by the Fourier transformation of Fig. $2 \mathrm{~b}$ also (a)

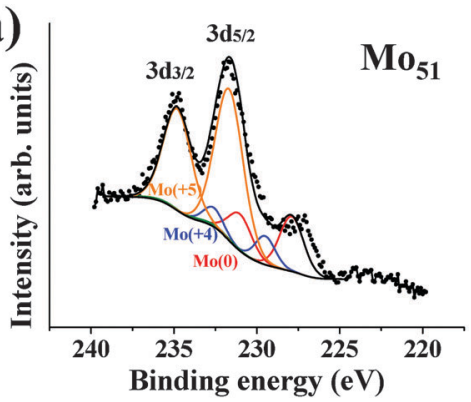

(c)

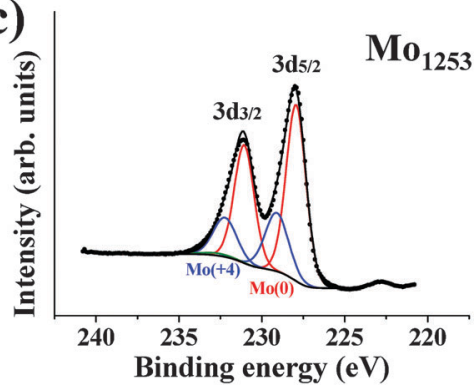

(b)

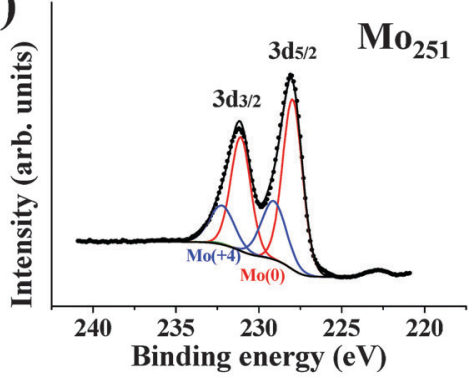

(d)

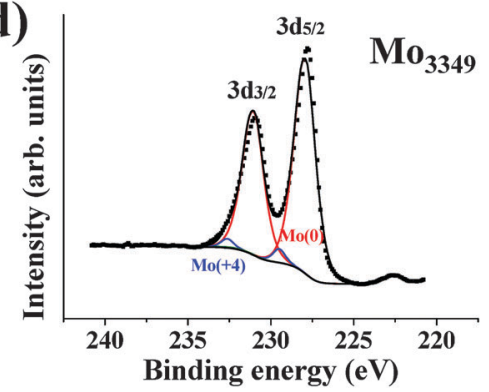

Fig. 1 In situ XPS spectra of (a) $\mathrm{Mo}_{51}$, (b) $\mathrm{MO}_{251}$, (c) $\mathrm{Mo}_{1253}$ and (d) $\mathrm{MO}_{3349}$ clusters deposited on HOPG. 

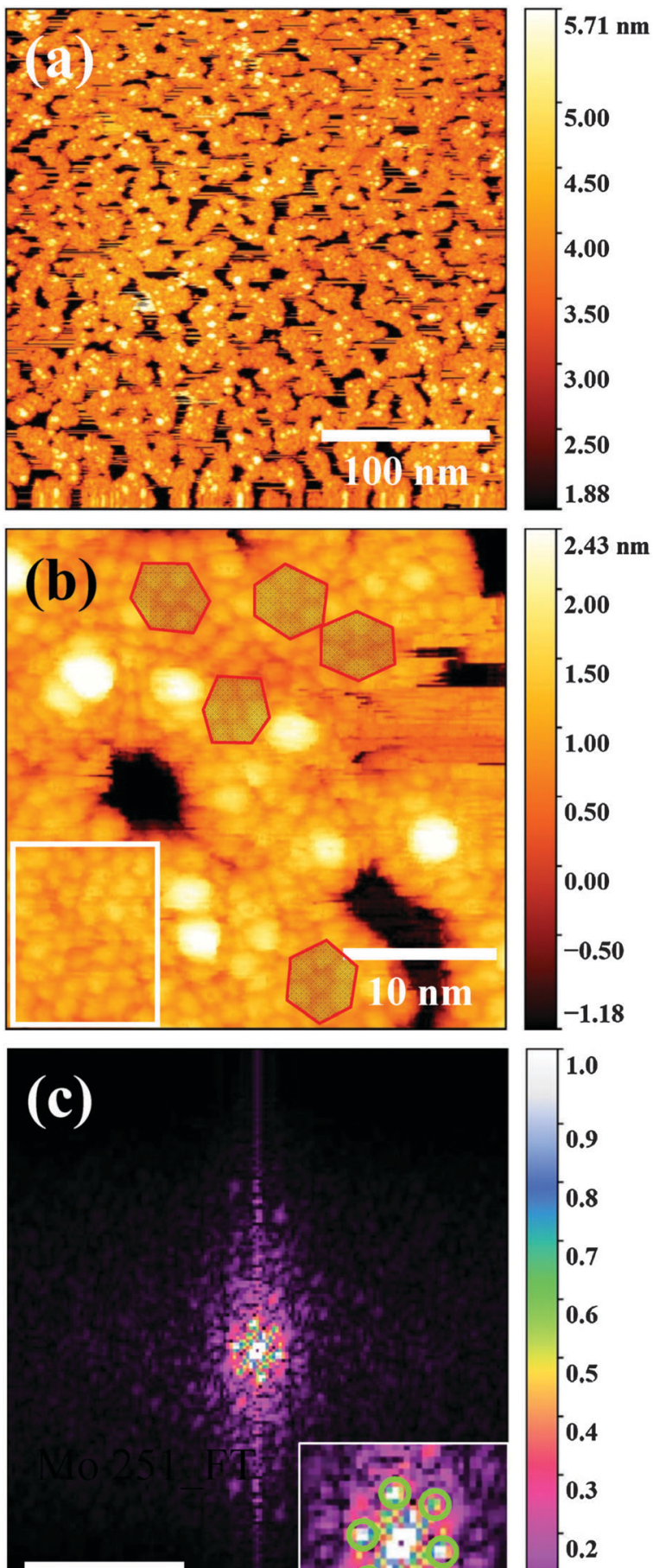

\section{$5 \mathrm{~nm}^{-1}$}

Fig. 2 In situ STM images of $\mathrm{MO}_{251}$ clusters deposited on HOPG at low coverage (tunneling parameters: $U=1.5 \mathrm{~V}, I=8 \mathrm{pA}$ ). Fig. $2 \mathrm{~b}$ shows a magnified view of (a). There is a local hexagonal order (marked). Figure $c$ shows a Fourier transformed pattern of the white rectangle in (b).

exhibits a hexagonal pattern, confirming the existence of an ordered structure of the $\mathrm{Mo}_{251}$ clusters (Fig. 2c).

Fig. 3 summarizes the STM results of the deposited Mo clusters with three different sizes (251, 1253, 3349 Mo atoms). The cluster coverage in each image corresponds to roughly one
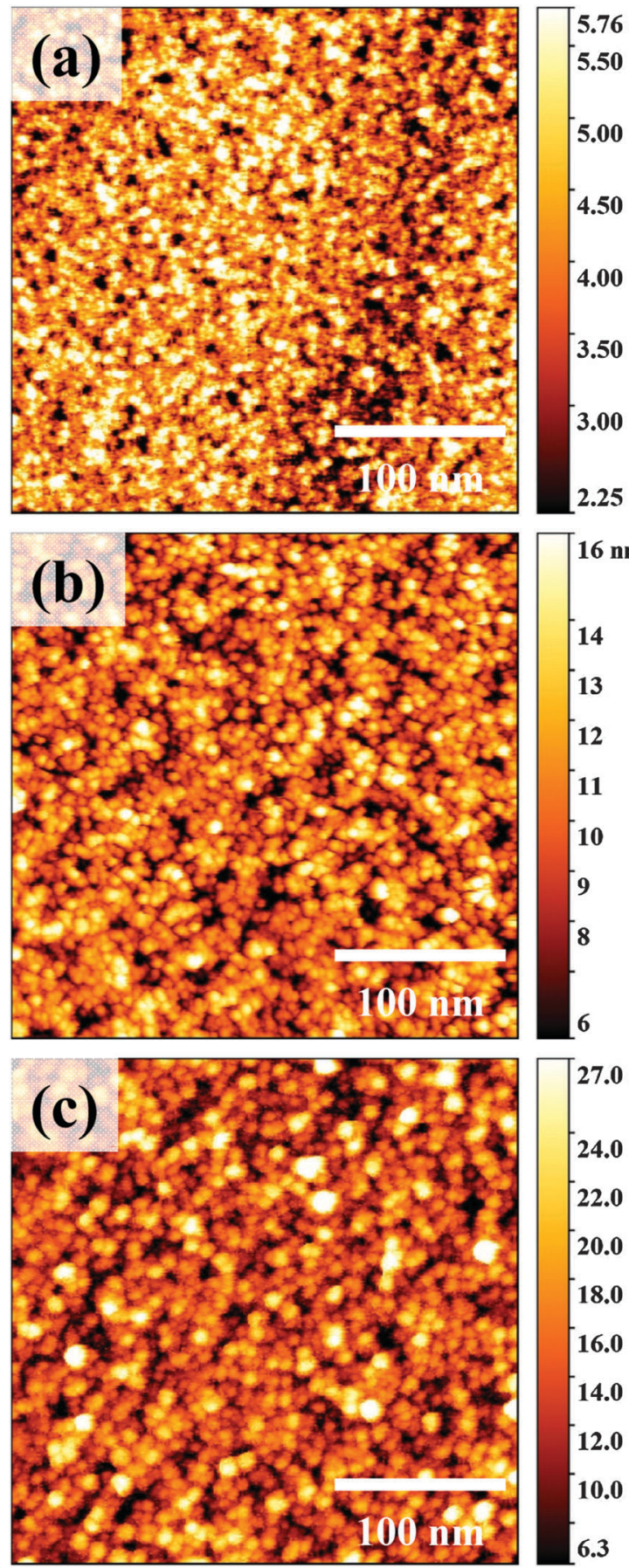

$27.0 \mathrm{~nm}$

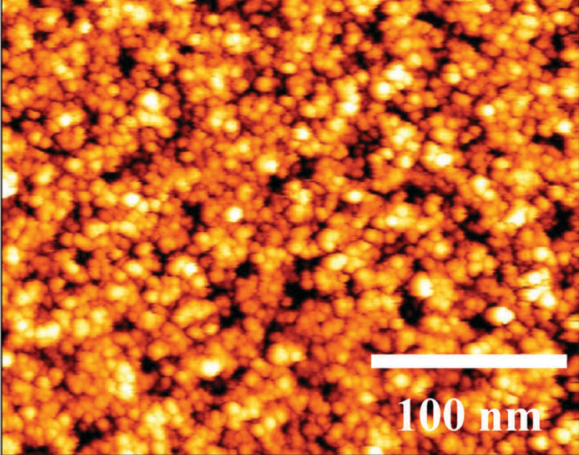

13

12

11

10

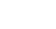

24.0

22.0

20.0

18.0

16.0

14.0

12.0

10.0

6.3

Fig. 3 In situ STM images of (a) $\mathrm{Mo}_{251}(U=1.5 \mathrm{~V}, I=6 \mathrm{pA})$, (b) $\mathrm{Mo}_{1253}$ $(U=2.0 \mathrm{~V}, I=5 \mathrm{pA})$ and $(\mathrm{c}) \mathrm{Mo}_{3349}(U=1.0 \mathrm{~V}, I=8 \mathrm{pA})$ clusters deposited on HOPG at a cluster coverage of $\sim$ monolayer.

monolayer. Each picture shows that the films consist of individual clusters with a cluster size of less than $5-10 \mathrm{~nm}$. The clusters do not fuse and survive as individual entities upon deposition. This is also applies to multilayers films (data not shown here).

\subsection{Results of the ex situ studies}

For several samples ex situ XPS spectra have been recorded to determine the degree of oxidation after exposure to air. 
(a)

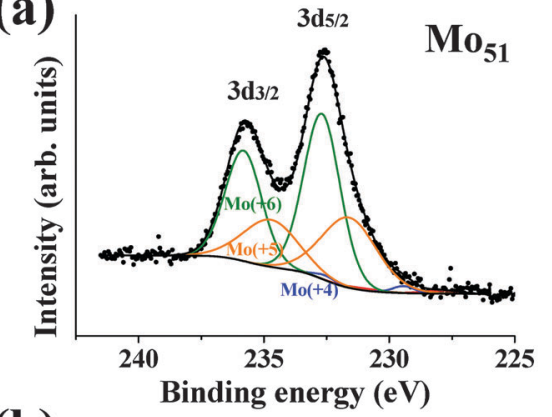

(b)

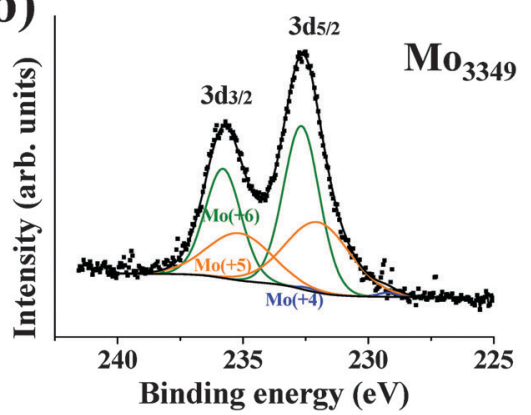

Fig. 4 XPS spectra of (a) $\mathrm{MO}_{51}$ and (b) $\mathrm{Mo}_{3349}$ clusters deposited on HOPG and exposed to air.

Fig. 4 displays XPS spectra of deposited $\mathrm{Mo}_{51}$ and $\mathrm{Mo}_{3349}$ clusters, which are the smallest and the largest clusters studied here.
For both clusters, $\operatorname{Mo}(\mathrm{vI})$ and $\mathrm{Mo}(\mathrm{v})$ are dominant species indicating almost complete oxidation even for the very large clusters. Clearly, air exposure has an dramatic effect on the cluster thin films. ${ }^{27}$ For all other impurities, only trace amounts have been detected.

Fig. 5 displays AFM images of $\mathrm{Mo}_{51}$ clusters deposited on HOPG and exposed to air. The coverage increases from sub monolayer ( $\mathrm{a}$ and $\mathrm{b}$ ) to a cluster monolayer (c) and finally to a multilayer film (d). At lowest coverage (a), individual clusters decorating either step edges or terraces can be identified. On the terraces, the clusters or islands of clusters are probably stabilized by point defects. ${ }^{28}$ With increasing cluster coverage (b), 2-D islands are observed. These islands exhibit no internal fine structure indicating that these relatively small clusters are fused. The heights of these islands can be measured quite accurately with the AFM. All islands are of similar height of about $2 \mathrm{~nm}$. This height is close to the calculated diameter of a single oxidized $\mathrm{Mo}_{51}$ cluster. This is also true for the almost closed monolayer (Fig. 5c).

It seems that the clusters agglomerate into 2-D islands. This can be explained by the following scenario. The clusters on the surface are highly mobile and form loosely bound 2-D islands. Within these islands the individual clusters survive as long as the high vacuum conditions are maintained. Even though the clusters are already partially oxidized under high vacuum conditions, exposure to air results in the oxidation of the clusters,
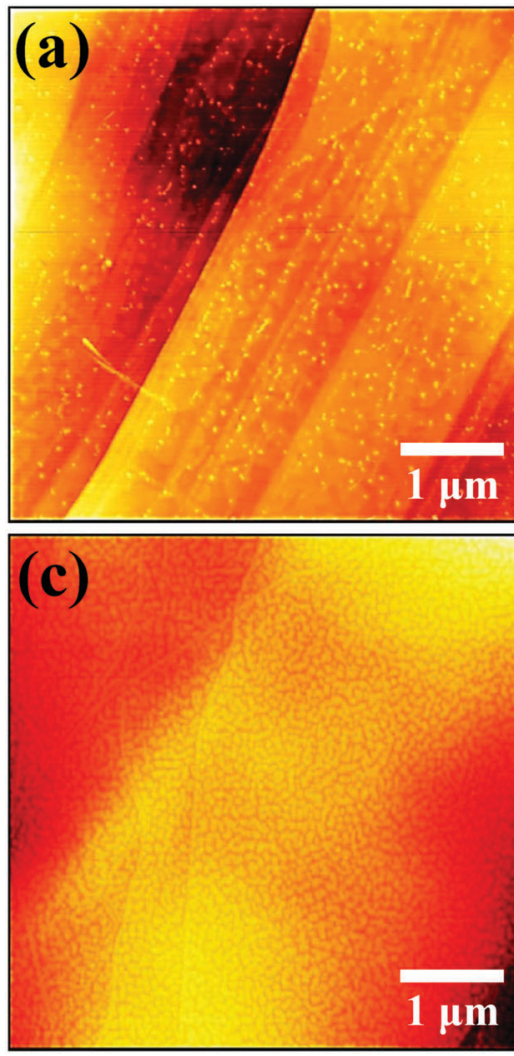
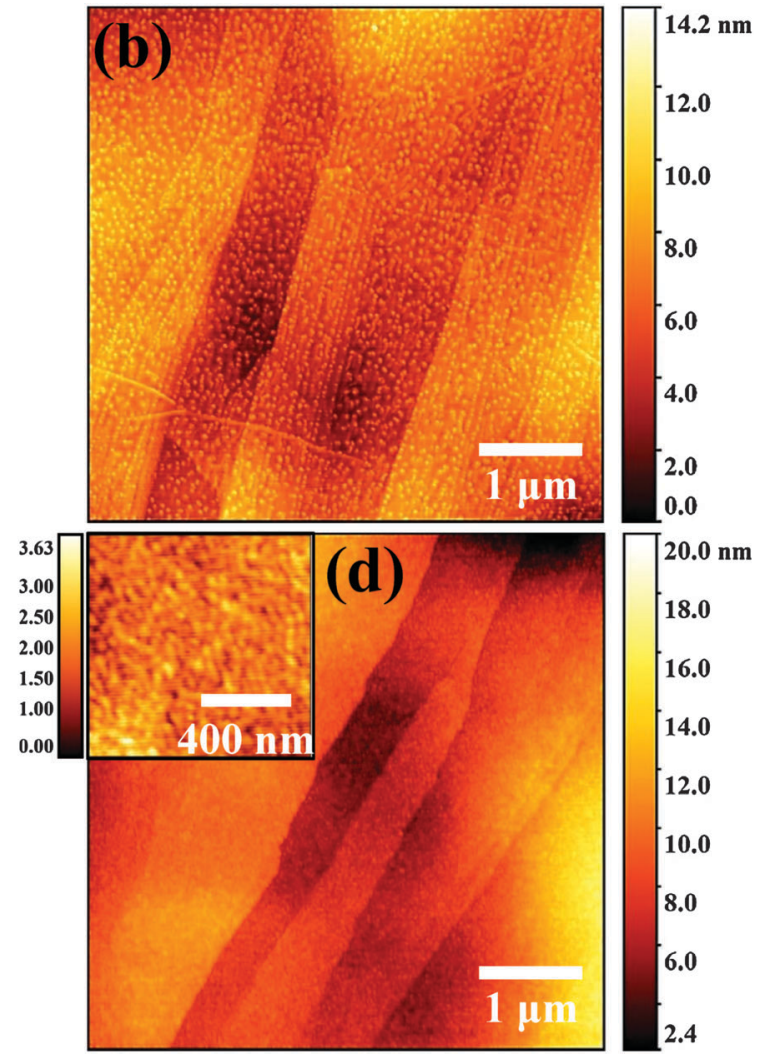

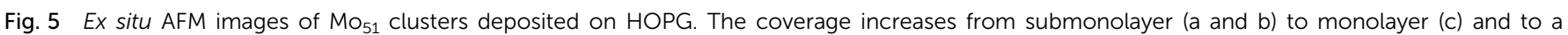
multilayer film (d). For (d) a magnified view is provided in the inset. 

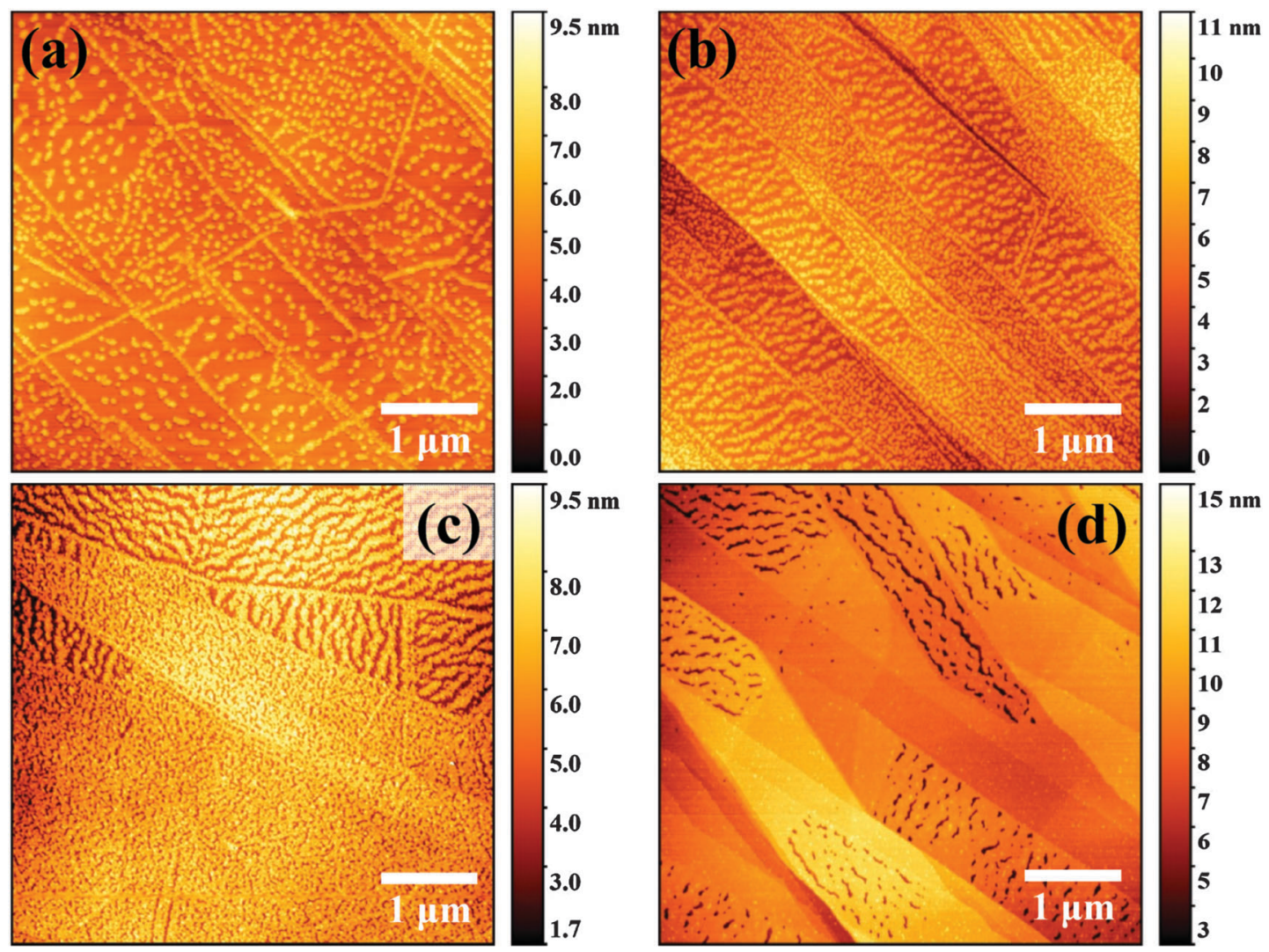

Fig. 6 AFM images of deposited $\mathrm{Mo}_{251}$ clusters. The coverage increases from (a) to (d). In (d) the coverage corresponds to a monolayer of clusters.

which is a quite exothermic process. This could results in the fusion of the neighboring clusters and a flat sheet of Mo oxide is formed. A clarification of the processes requires the recording of in situ STM pictures. As already mentioned, imaging in situ STM pictures of the surface consisting of $\mathrm{Mo}_{51}$ has not been successful so far. It is worth mentioning, that such a monolayer film of a metal-oxide on HOPG cannot be prepared by conventional thin film deposition techniques. HOPG is a weakly interacting van der Waals surface on which atom vapor deposition generally leads to the formation of 3D islands. In contrast to a smooth and closed monolayer, such a film is rough and has an open structure.

At higher coverage above a monolayer the surface becomes rather rough and "dotted". (Fig. 5d, inset). The surface of the cluster monolayer is not a smooth van der Waals surface and interacts much stronger with the clusters. Accordingly, clusters on the monolayer have a lower mobility. The clusters stick to their landing sites and are randomly distributed over the surface. The data indicate that these clusters survive as individual entities.

Fig. 6 displays an analogous series of ex situ AFM pictures recorded for $\mathrm{Mo}_{251}$ clusters. From (a) to (d), the coverage increases up to the closure of a monolayer. Fig. $6 \mathrm{~d}$ displays a surprisingly smooth and perfect cluster monolayer covering the HOPG surface. The growth mode seems to be almost identical to that of $\mathrm{Mo}_{51}$. However, a close inspection of the AFM images reveals a dissimilarity (Fig. 7). In the case of $\mathrm{Mo}_{51}$, the periphery of the 2-D islands is smooth, whereas for $\mathrm{Mo}_{251}$ it is rather frayed. We take that as an indication that for $\mathrm{Mo}_{251}$ the 2-D islands consist of individual particles rather than a fused compact oxide layer. The comparison with the in situ STM image of the same sample shows that the $\mathbf{M o}_{251}$ clusters survive as individual entities upon deposition and even after air exposure.

In contrast to the case of the small clusters, for the medium sizes clusters so-called "pylons" appear at higher coverage (Fig. 8a). The "pylons" are relatively large 3-D objects with a diameter of $\sim 200 \mathrm{~nm}$. They have a broad and approximately symmetric size distribution (Fig. 8b). Such structures are the result of ripening processes. There are two different mechanisms which might occur in the case of deposited clusters, namely Ostwald and Smolukowski ripening. ${ }^{29}$ In Ostwald ripening, large clusters grow on the expense of small clusters. ${ }^{29}$ The small particles loose monomers because of their higher vapor pressure, while the larger particles collect monomers. This ripening process would result in the formation of large massive particles. The pylons consist of individual particles, which is obvious for the $\mathrm{Mo}_{1253}$ clusters (Fig. 9). Therefore, Ostwald ripening cannot explain our observations. The second possible process is Smolukowski ripening. Here, the clusters move as a whole and coalesce into larger 3-D structures with a rather symmetric size distribution. ${ }^{30}$ For clusters of common metals, Smolukowski ripening results in the formation of large bulklike particles. Again, this does not fit to our observations, because within each pylon individual clusters are still recognizable. Instead, the Mo clusters seemed to be glued together such 

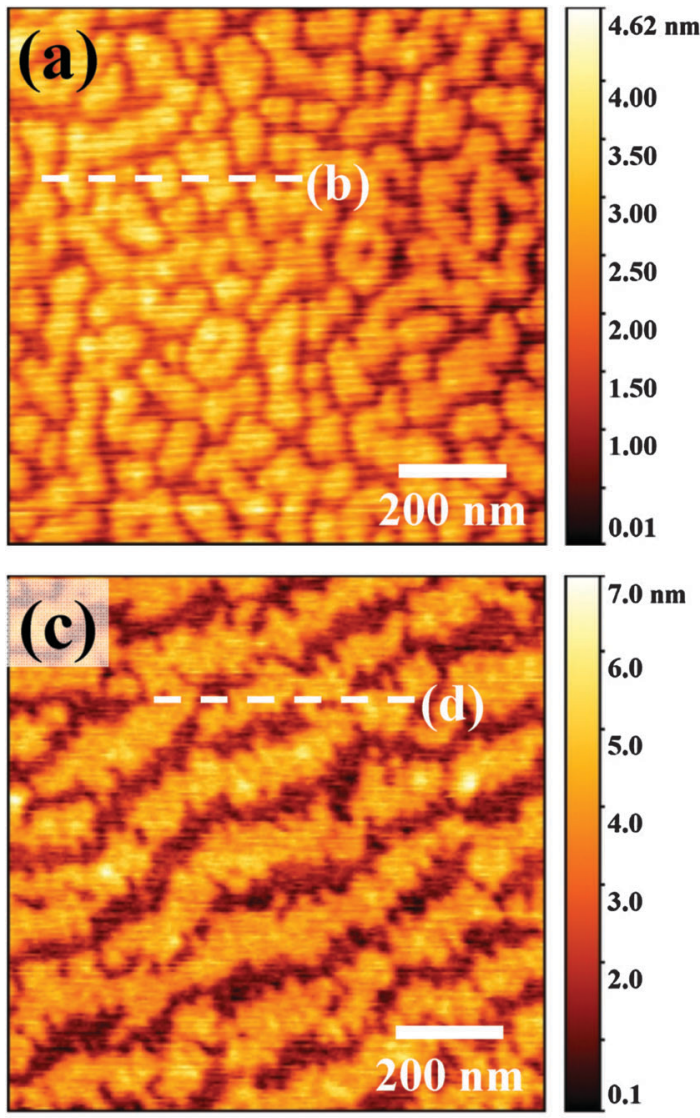

(b)

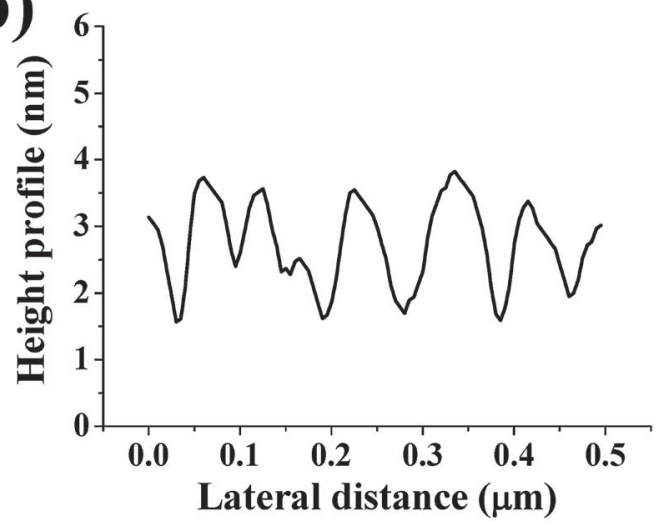

(d)

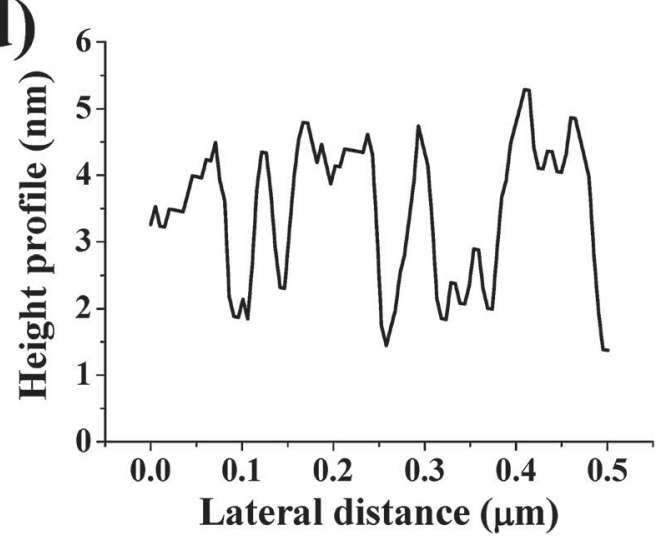

Fig. 7 In (a) and (c) magnified views of Fig. $2 c$ and $3 c$ are displayed, respectively. In (b) and (d) height profiles of (a) and (c) are shown, respectively.

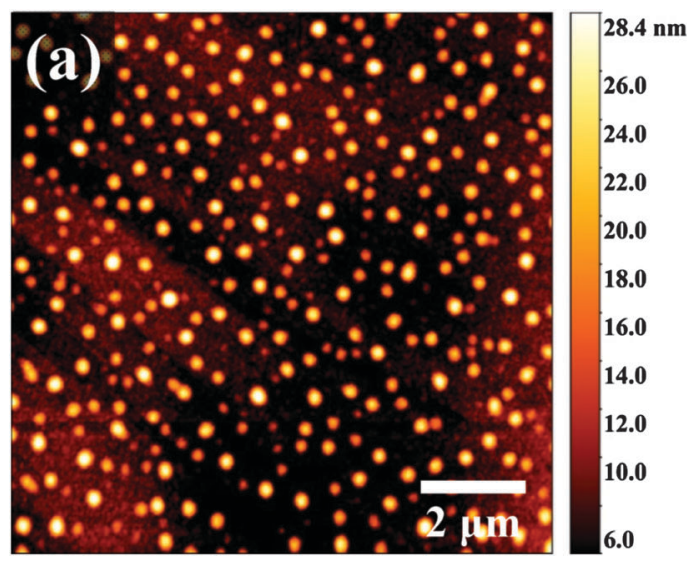

(b)

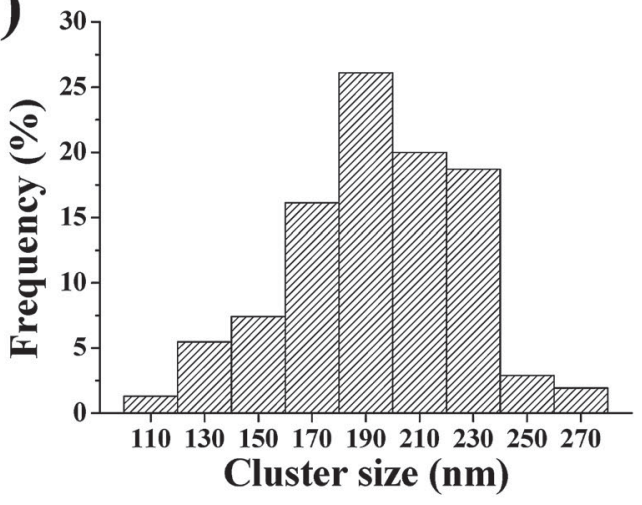

Fig. 8 (a) Ex situ AFM image of $M_{251}$ clusters deposited on HOPG at high coverage. (b) Result of the statistical analysis of the pylon size. 310 pylons were analyzed.

as sand grains in sandstone (Fig. 9d). Our observations can be best explained by incomplete Smolukowski ripening. The particles agglomerate, but because of the high melting point of the material the process is incomplete. The formation of the pylons corresponds to a rather huge reorganization of the film. For this, quite some energy is required. It seems plausible that the ripening process is driven by the oxidation taking place after exposure to air, because only this process provides enough energy. It is worth mentioning that such pylon structures have not been observed in our in situ STM studies.

Pylons are not observed for large $\left(\mathrm{Mo}_{3349}\right.$, see below) and for small $\left(\mathrm{Mo}_{51}\right)$ clusters. The large clusters have a very low mobility, which explains that there are no pylons. For the smallest clusters, there are no pylons because these clusters immediately coalesce either already under high vacuum conditions or after exposure to air. Here, oxidation is very fast and 

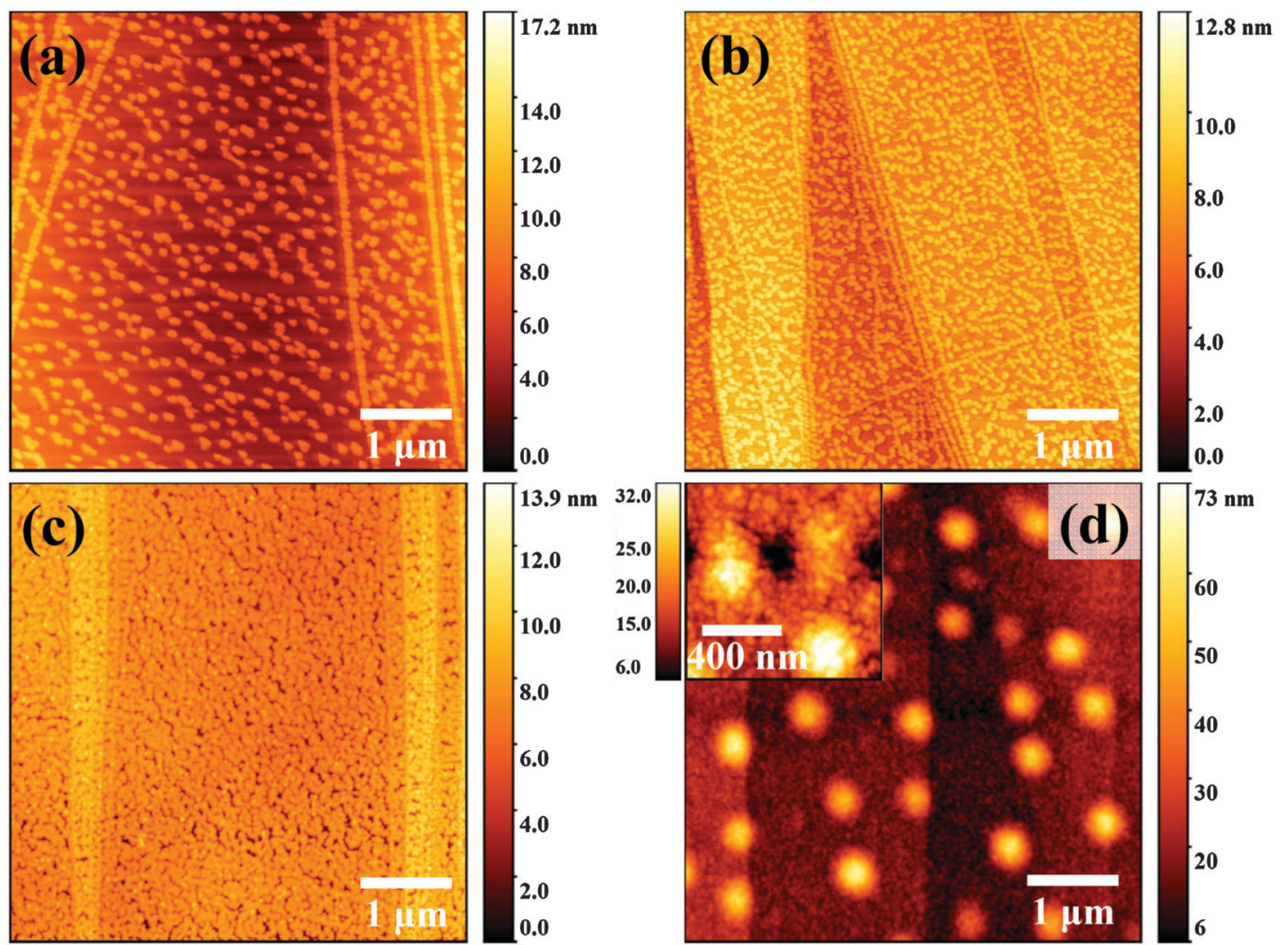

Fig. 9 Ex situ AFM images of $\mathrm{Mo}_{1253}$ clusters deposited on HOPG. The coverage increases from (a) to (d). In (d) a magnified view of a "pylon" in shown in the inset.
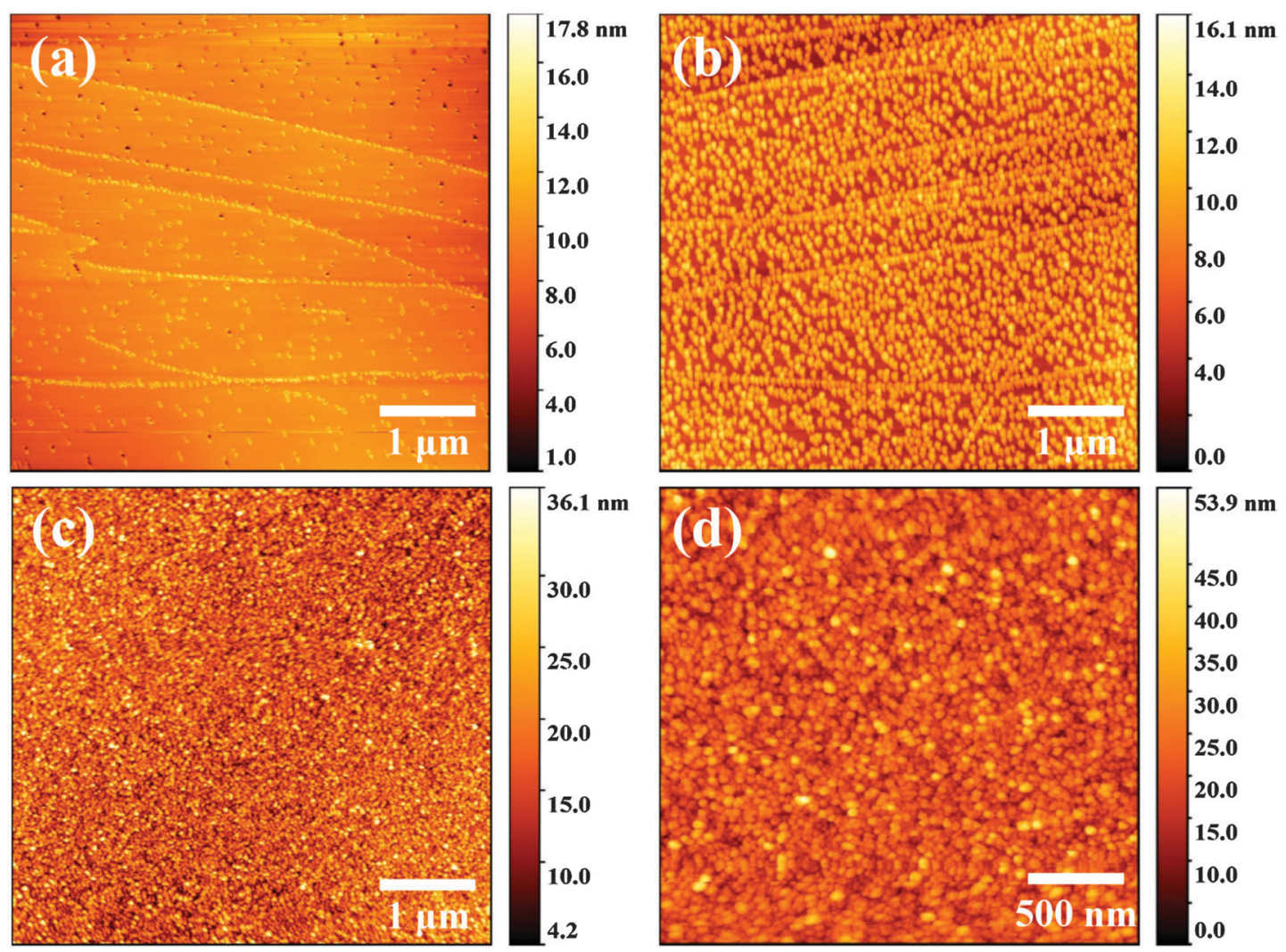

Fig. 10 Ex situ AFM images of $\mathrm{MO}_{3349}$ clusters deposited on HOPG. From (a) to (c) the coverage increases. (d) Is magnified view of (c). 
the whole film converts in a $\mathrm{MoO}_{3}$ sheet. There is no time for pylon formation. Only for the medium size particles oxidation is slow enough for the ripening process. Of course, these are all speculations. Further experiments, namely the recording of more in situ STM pictures, are required to clarify the processes determining the structures of the cluster thin films.

Thin films of $\mathrm{Mo}_{1253}$ clusters show a behavior very similar to that of $\mathrm{Mo}_{251}$. At low coverage, 2-D islands are formed and which grow into an almost perfect monolayer (Fig. 9a-c). At high coverage pylons are observed (Fig. 9d), which consist of individual particles (Fig. 9d inset).

For large clusters $\left(\mathrm{Mo}_{3349}\right)$ a different pattern is observed (Fig. 10). At low coverage the cluster are distributed randomly over the surface (Fig. 10a and b). Some decorate step edges, but there are much more clusters on the terraces than in the case of the smaller clusters. This can be explained by the low mobility of these big particles, which tend to stick to their landing sites. At zero mobility, there would be no preference for the step edges and the distribution would be completely random. This is not the case for the $\mathrm{Mo}_{3349}$ clusters indicating that there is still some mobility. There is no formation of 2-D islands and no indication for the formation of a monolayer. Instead, the film surface exhibits an increasing roughness. Again, this can be explained by a low mobility because the formation of a smooth monolayer requires a high mobility of the clusters on the substrate. There is no coalescence and even at higher coverage (Fig. 10c and d) individual clusters can be discerned in the AFM pictures.

\section{Conclusion}

Size-selected bare Mo clusters in the size range between 50 and 3500 atoms were deposited on highly ordered pyrolytic graphite (HOPG) and exposed to air. HOPG is a van der Waals surface and it is known to interact very weakly with deposited metal atoms and clusters. Accordingly, we expected to observe three dimensional growth modes similar to the case of Mo atoms, ${ }^{26}$ but this is not what we have found. At low coverage, small and medium sizes clusters form a continuous almost perfect monolayer, which consists of individual clusters. Deposition of additional clusters on top of the monolayer yields either a rugged granular structure or protruded and separate pylons $\left(\mathrm{Mo}_{251}\right.$ and $\left.\mathrm{Mo}_{1253}\right)$. For $\mathrm{Mo}_{3343}$, no monolayer is formed. Instead, the clusters stick to their landing sites and the surface roughness increases with increasing coverage.

The formation of smooth monolayers on a van der Waals surface and the observation of "pylons" are new and unique to cluster thin films. These properties may be useful for applications in photocatalysis and for optoelectronic devices. Especially the monolayers might be interesting for certain applications, because they have an adjustable and extremely homogenous thickness. Cluster deposition works even on surfaces, which are difficult to coat with conventional techniques. In addition to the studies on the growth modes, we show that medium-sized and large clusters do not coalesce. They are building blocks of new cluster materials.

\section{Acknowledgements}

Support by German science foundation (Grant No. GA 389/15-1) is gratefully acknowledged. This work was supported by the National Research Council of Science and Technology (NST) through Degree and Research Center (DRC) Program (2014).

\section{References}

1 E. Fortunato, P. Barquinha and R. Martins, Adv. Mater., 2012, 24, 2945-2986.

2 K. L. Choy, Prog. Mater. Sci., 2003, 48, 57-170.

3 A. Fujishima, X. T. Zhang and D. A. Tryk, Surf. Sci. Rep., 2008, 63, 515-582.

4 G. Renaud, R. Lazzari and F. Leroy, Surf. Sci. Rep., 2009, 64, 255-380.

5 S. Panigrahi, S. Basu, S. Praharaj, S. Pande, S. Jana, A. Pal, S. K. Ghosh and T. Pal, J. Phys. Chem. C, 2007, 111, 4596-4605.

6 M. Haruta, Gold Bull., 2004, 37, 27-36.

7 A. S. K. Hashmi and G. J. Hutchings, Angew. Chem., Int. Ed., 2006, 45, 7896-7936.

8 M. Haruta and M. Date, Appl. Catal., A, 2001, 222, 427-437.

9 D. Stolcic, M. Fischer, G. Ganteför, Y. D. Kim, Q. Sun and P. Jena, J. Am. Chem. Soc., 2003, 125, 2848-2849.

10 A. D. Yoffe, Adv. Phys., 2001, 50, 1-208.

11 G. Schmid, Chem. Soc. Rev., 2008, 37, 1909-1930.

12 Y. J. Ko, A. Shakya, H. P. Wang, A. Grubisic, W. J. Zheng, M. Gotz, G. Ganteför, K. H. Bowen, P. Jena and B. Kiran, J. Chem. Phys., 2010, 133, 124308.

13 B. Kiran, P. Jena, X. Li, A. Grubisic, S. T. Stokes, G. F. Ganteför, K. H. Bowen, R. Burgert and H. Schnockel, Phys. Rev. Lett., 2007, 98, 256802.

14 H. C. Weissker, H. B. Escobar, V. D. Thanthirige, K. Kwak, D. Lee, G. Ramakrishna, R. L. Whetten and X. LopezLozano, Nat. Commun., 2014, 5, 3785.

15 B. Yoon, V. M. Akulin, P. Cahuzac, F. Carlier, M. de Frutos, A. Masson, C. Mory, C. Colliex and C. Brechignac, Surf. Sci., 1999, 443, 76-88.

16 A. Majumdar, M. Ganeva, D. Kopp, D. Datta, P. Mishra, S. Bhattacharayya, D. Ghose and R. Hippler, Vacuum, 2008, 83, 719-723.

17 R. P. Andres, J. D. Bielefeld, J. I. Henderson, D. B. Janes, V. R. Kolagunta, C. P. Kubiak, W. J. Mahoney and R. G. Osifchin, Science, 1996, 273, 1690-1693.

18 T. M. Bernhardt, B. Stegemann, B. Kaiser and K. Rademann, Angew. Chem., Int. Ed., 2003, 42, 199-202.

19 R. E. Smalley and B. I. Yakobson, Solid State Commun., 1998, 107, 597-606.

20 M. Núñez-Regueiro, L. Marques, J. L. Hodeau, O. Béthoux and M. Perroux, Phys. Rev. Lett., 1995, 74, 278-281.

21 M. Grass, D. Fischer, M. Mathes, G. Ganteför and P. Nielaba, Appl. Phys. Lett., 2002, 81, 3810-3812.

22 X. Li, K. Wepasnick, X. Tang, D. H. Fairbrother, K. H. Bowen, A. Dollinger, C. H. Strobel, J. Huber, T. Mangler, Y. Luo, S. Proch and G. Gantefoer, J. Appl. Phys., 2014, 115, 104304. 
23 M. Willis, M. Gotz, A. K. Kandalam, G. F. Ganteför and P. Jena, Angew. Chem., Int. Ed., 2010, 49, 8966-8970.

24 X. Li, A. Grubisic, S. T. Stokes, J. Cordes, G. F. Ganteför, K. H. Bowen, B. Kiran, M. Willis, P. Jena, R. Burgert and H. Schnockel, Science, 2007, 315, 356-358.

25 C. Berkdemir, A. W. Castleman and J. O. Sofo, Phys. Chem. Chem. Phys., 2012, 14, 9642-9653.

26 X. Xu, H. Permana, Y. Lu and K. Y. S. Ng, Surf. Sci., 1995, 325, 285-293.
27 J. F. Moulder and J. Chastain, Handbook of X-ray Photoelectron Spectroscopy: A Reference Book of Standard Spectra for Identification and Interpretation of XPS Data, Physical Electronics, 1995.

28 I. Lopez-Salido, D. C. Lim and Y. D. Kim, Surf. Sci., 2005, 588, 6-18.

29 P. Sahu and B. L. V. Prasad, Langmuir, 2014, 30, 10143-10150.

30 C. R. Stoldt, C. J. Jenks, P. A. Thiel, A. M. Cadilhe and J. W. Evans, J. Chem. Phys., 1999, 111, 5157-5166. 